\title{
Varying impacts of China's coal ban
}

Shonali Pachauri, Senior Research Scholar, Energy Program, International Institute for Applied Systems Analysis (IIASA), Schlossplatz 1, A-2361 Laxenburg, Austria. Email: pachauri@iiasa.ac.at

Policies that encourage a shift away from solid fuels can improve public health, welfare and the environment. However, impacts and their distribution are rarely evaluated. Now research shows that benefits to home comfort, indoor air quality and well-being from China's coal-to-electricity programme vary across rich and poor districts.

Reducing coal burning in China, where over one-third of homes continue to rely on coal stoves for heating and cookingi, could eliminate a chief source of hazardous fine particulate matter, which is a major source of air pollution and is responsible for a significant proportion of illness and death globallyii. In addition to public health benefits, the adoption of alternative fuel sources could reduce the drudgery involved in acquiring, storing and using coal, improve home comfort and mitigate climate change. In response to these environmental and health concerns, a 'coal-to-electricity' programme that subsidizes electricity and electric heat pumps and bans coal is being rolled out village by village in Northern China. It has already been implemented in 3,700 villages throughout the Beijing municipal region. Writing in Nature Energy, Christopher Barrington-Leigh, from McGill University, and co-authors ${ }^{\text {iii }}$ take advantage of the phased implementation of this programme to compare villages that have benefitted from the programme and those that have not, thus allowing them to assess the impacts of this policy in a natural experiment.

Policies encouraging alternative fuel sources are rarely targeted to the poor, and few analyses attempt to understand policy impacts on different population subgroups. Barrington-Leigh et al. surveyed households in treated and untreated villages in three districts of varying affluence levels surrounding Beijing to determine whether the programme's impacts on energy use for heating, household economics and welfare, and the environment vary based on socioeconomic conditions. Such analyses are important to understand whether diverse regions and population subgroups benefit equitably from policies, and to inform efforts to better target policies to be more effective and ease disparities.

The results show that coal use has been eliminated in the treated villages in wealthier districts, and significantly lowered in a treated village in a less affluent district. The evaluation also clearly reveals a general shift from less efficient electrical heating technologies to more efficient ones, which are also considered safer and more convenient to use. In addition, comfort in homes appears to have improved in treated villages, with higher use of heating, warmer indoor temperatures and a greater fraction of house area and rooms heated - at least in the wealthier districts surveyed. Within the less affluent district surveyed, wealthier households are less likely to spend money heating with coal. However, poorer households struggle to comply with the coal ban despite subsidies, because the cost of heating with electric heat pumps is still unaffordable. This implies that the existing subsidies are insufficient to enable a complete transition away from coal by the poor. Perhaps due to these financial constraints that prevent coal from being completely abandoned in poorer households, 
improvements in indoor air quality (as measured by concentrations of fine particulate matter) in treated villages were only observed in more affluent districts. This echoes the results of other recent assessments of the programme that also concluded that while private health benefits from improvements to indoor air quality may be low as poor households continue to use coal, improvements to outdoor air quality have resulted in substantial social benefits regionallyiv, .

In addition to general questions related to methods used to heat each room in the home and heating expenditures, Barrington-Leigh et al. also included subjective questions on overall satisfaction with life, living conditions and household income that are seldom included in such policy evaluation studies. They found that shifts in heating behaviours in more affluent districts appear to be accompanied by improvements in satisfaction with life and living conditions. In the less affluent district, however, self-reported perception of life satisfaction was relatively lower in the treated village compared with the untreated village, suggesting that the programme might have had negative welfare impacts in households that could not afford to shift to the more expensive alternatives. This raises concerns for the scalability and transferability of the programme, because even if overall benefits have been positive, impacts seem to be uneven across socioeconomic contexts.

Taken together, these results suggest that the combination of a coal ban with the provision of more efficient alternative electric heating technologies and public subsidies for equipment and energy have been partially successful in changing heating behaviours and improving perceived wellbeing, at least in homes in more affluent villages. This is encouraging news for household energy transition policies that seek to ban harmful solid fuel burning to improve air quality, public health and welfare. At the same time, this programme evaluation provides important insights for course corrections to the coal-to-electricity programme as well as lessons for other countries or regions that might be considering similar policies. For example, the fact that impacts were less positive in less affluent villages suggests that better designed and more targeted support might be required, with a particular focus on poor households and/or regions, to ensure that inequalities are not exacerbated. While administratively more burdensome, targeting is increasingly being implemented in other nations, for instance in India's gas for cooking promotion programme that specifically targets women in poor households (http://www.pmujiwalayojana.com/).

Analyses that evaluate large-scale, real-world initiatives, such as the one highlighted in this study by Barrington-Leigh and co-authors ${ }^{\mathrm{iii}}$, need to become a routine part of programme rollouts. These should include a better quantification of benefits, costs and impacts for different population groups and diverse geographic regions. Understanding how and why benefits and costs might vary across diverse regions and groups can aid policymakers in formulating fairer policies. Regular evaluations could also reveal seasonal variations and longer-term impacts that could support the design of more effective policies and programmes. 


\section{References}

i Duan et al. Applied Energy, 136:692-703, (2014).

ii Global Burden of Disease Collaborative Network (2017) IHME Global Burden of Disease Study 2016 (GBD 2016) results (Institute for Health Metrics and Evaluation, Seattle).

iii Barrington-Leigh et al. Nature Energy 4, XX-XX (2019).

iv Zhang et al. Energy Policy, 127: 165-178, (2019).

$\checkmark$ Liu et al. Environment International, 125: 236-244, (2019). 